\title{
On a half-discrete reverse Mulholland-type inequality and an extension
}

Tuo Liu', Bicheng Yang ${ }^{2}$ and Leping $\mathrm{He}^{1 *}$

\section{"Correspondence:}

jdheleping@163.com

${ }^{1}$ College of Mathematics and

Statistics, Jishou University, Hunan,

Jishou 416000, P.R. China

Full list of author information is

available at the end of the article

\begin{abstract}
By using the way of weight functions and the Hermite-Hadamard inequality, a half-discrete reverse Mulholland-type inequality with a best constant factor is given. The extension with multi-parameters, the equivalent forms as well as the relating homogeneous inequalities are also considered.

MSC: $26 \mathrm{D} 15$
\end{abstract}

Keywords: Mulholland-type inequality; weight function; equivalent form; reverse

\section{Introduction}

Assuming that $f, g \in L^{2}\left(R_{+}\right),\|f\|=\left\{\int_{0}^{\infty} f^{2}(x) d x\right\}^{\frac{1}{2}}>0,\|g\|>0$, we have the following Hilbert integral inequality (cf. [1]):

$$
\int_{0}^{\infty} \int_{0}^{\infty} \frac{f(x) g(y)}{x+y} d x d y<\pi\|f\|\|g\|
$$

where the constant factor $\pi$ is best possible. If $a=\left\{a_{n}\right\}_{n=1}^{\infty}, b=\left\{b_{n}\right\}_{n=1}^{\infty} \in l^{2},\|a\|=$ $\left\{\sum_{n=1}^{\infty} a_{n}^{2}\right\}^{\frac{1}{2}}>0,\|b\|>0$, then we still have the following discrete Hilbert inequality:

$$
\sum_{m=1}^{\infty} \sum_{n=1}^{\infty} \frac{a_{m} b_{n}}{m+n}<\pi\|a\|\|b\|
$$

with the same best constant factor $\pi$. Inequalities (1) and (2) are important in analysis and its applications $(c f$. [2-4]). Also we have the following Mulholland inequality with the same best constant factor $\pi(c f .[1,5])$ :

$$
\sum_{m=2}^{\infty} \sum_{n=2}^{\infty} \frac{a_{m} b_{n}}{\ln m n}<\pi\left\{\sum_{m=2}^{\infty} m a_{m}^{2} \sum_{n=2}^{\infty} n b_{n}^{2}\right\}^{\frac{1}{2}} .
$$

In 1998, by introducing an independent parameter $\lambda \in(0,1]$, Yang [6] gave an extension of (1). For generalizing the results from [6], Yang [7] gave some best extensions of (1) and (2) as follows. If $p>1, \frac{1}{p}+\frac{1}{q}=1, \lambda_{1}+\lambda_{2}=\lambda, k_{\lambda}(x, y)$ is a non-negative homogeneous function of degree $-\lambda, k\left(\lambda_{1}\right)=\int_{0}^{\infty} k_{\lambda}(t, 1) t^{\lambda_{1}-1} d t \in R_{+}, \phi(x)=x^{p\left(1-\lambda_{1}\right)-1}, \psi(x)=$ $x^{q\left(1-\lambda_{2}\right)-1}, f(\geq 0) \in L_{p, \phi}\left(R_{+}\right)=\left\{f \mid\|f\|_{p, \phi}:=\left\{\int_{0}^{\infty} \phi(x)|f(x)|^{p} d x\right\}^{\frac{1}{p}}<\infty\right\}, g(\geq 0) \in L_{q, \psi}\left(R_{+}\right)$, 
$\|f\|_{p, \phi},\|g\|_{q, \psi}>0$, then

$$
\int_{0}^{\infty} \int_{0}^{\infty} k_{\lambda}(x, y) f(x) g(y) d x d y<k\left(\lambda_{1}\right)\|f\|_{p, \phi}\|g\|_{q, \psi},
$$

where the constant factor $k\left(\lambda_{1}\right)$ is best possible. Moreover, if $k_{\lambda}(x, y)$ is finite and $k_{\lambda}(x, y) x^{\lambda_{1}-1}\left(k_{\lambda}(x, y) y^{\lambda_{2}-1}\right)$ is strict decreasing for $x>0(y>0)$, then for $a_{m}, b_{n} \geq 0, a=$ $\left\{a_{m}\right\}_{m=1}^{\infty} \in l_{p, \phi}=\left\{a \mid\|a\|_{p, \phi}:=\left\{\sum_{n=1}^{\infty} \phi(n)\left|a_{n}\right|^{p}\right\}^{\frac{1}{p}}<\infty\right\}, b=\left\{b_{n}\right\}_{n=1}^{\infty} \in l_{q, \psi},\|a\|_{p, \phi},\|b\|_{q, \psi}>0$, we have

$$
\sum_{m=1}^{\infty} \sum_{n=1}^{\infty} k_{\lambda}(m, n) a_{m} b_{n}<k\left(\lambda_{1}\right)\|a\|_{p, \phi}\|b\|_{q, \psi}
$$

with the same best constant factor $k\left(\lambda_{1}\right)$. Clearly, for $p=q=2, \lambda=1, k_{1}(x, y)=\frac{1}{x+y}, \lambda_{1}=$ $\lambda_{2}=\frac{1}{2}$, (4) reduces to (1), while (5) reduces to (2).

Some other results including the reverse Hilbert-type inequalities are provided by [816]. On half-discrete Hilbert-type inequalities with the non-homogeneous kernels, Hardy et al. provided a few results in Theorem 351 of [1]. But they did not prove that the constant factors in the inequalities are best possible. However, Yang [17] gave a result by introducing an interval variable and proved that the constant factor is best possible. Recently, Yang [18] gave a half-discrete Hilbert inequality with multi-parameters, and [19] gave the following half-discrete reverse Hilbert-type inequality with the best constant factor 4: For $0<p<1$, $\frac{1}{p}+\frac{1}{q}=1$, we have $\theta_{1}(x) \in(0,1)$, and

$$
\begin{aligned}
& \int_{0}^{\infty} f(x) \sum_{n=1}^{\infty} \min \{x, n\} a_{n} d x \\
& \quad>4\left\{\int_{0}^{\infty} \frac{1-\theta_{1}(x)}{x^{1-\frac{3 p}{2}}} f^{p}(x) d x\right\}^{\frac{1}{p}}\left\{\sum_{n=1}^{\infty} \frac{a_{n}^{q}}{n^{1-\frac{3 q}{2}}}\right\}^{\frac{1}{q}} .
\end{aligned}
$$

In this paper, by using the way of weight functions and the Hermite-Hadamard inequality, a half-discrete reverse Mulholland-type inequality similar to (6) is given as follows:

$$
\begin{aligned}
& \int_{0}^{\infty} f(x) \sum_{n=1}^{\infty} \frac{a_{n}}{\ln e\left(n+\frac{1}{2}\right)^{x}} d x \\
& \quad>\pi\left\{\int_{0}^{\infty} \frac{1-\theta_{1}(x)}{x^{1-\frac{p}{2}}} f^{p}(x) d x\right\}^{\frac{1}{p}}\left\{\sum_{n=1}^{\infty} \frac{\left(n+\frac{1}{2}\right)^{q-1} a_{n}^{q}}{\ln ^{1-\frac{q}{2}}\left(n+\frac{1}{2}\right)}\right\}^{\frac{1}{q}} .
\end{aligned}
$$

Moreover, a best extension of (7) with multi-parameters, the equivalent forms and the relating homogeneous inequalities are considered.

\section{Some lemmas}

Lemma 1 If $0<\lambda \leq 2, \alpha \geq \frac{1}{2}$, setting weight functions $\omega(n)$ and $\varpi(x)$ as follows:

$$
\omega(n):=\ln ^{\frac{\lambda}{2}}(n+\alpha) \int_{0}^{\infty} \frac{1}{\ln ^{\lambda} e(n+\alpha)^{x}} x^{\frac{\lambda}{2}-1} d x, \quad n \in \mathbf{N},
$$




$$
\varpi(x):=x^{\frac{\lambda}{2}} \sum_{n=1}^{\infty} \frac{\ln ^{\frac{\lambda}{2}-1}(n+\alpha)}{(n+\alpha) \ln ^{\lambda} e(n+\alpha)^{x}}, \quad x \in(0, \infty),
$$

we have

$$
B\left(\frac{\lambda}{2}, \frac{\lambda}{2}\right)\left(1-\theta_{\lambda}(x)\right)<\varpi(x)<\omega(n)=B\left(\frac{\lambda}{2}, \frac{\lambda}{2}\right),
$$

where

$$
\theta_{\lambda}(x)=\frac{1}{B\left(\frac{\lambda}{2}, \frac{\lambda}{2}\right)} \int_{0}^{x \ln (1+\alpha)} \frac{t^{(\lambda / 2)-1}}{(1+t)^{\lambda}} d t \in(0,1)
$$

satisfying $\theta_{\lambda}(x)=O\left(x^{\frac{\lambda}{2}}\right)$.

Proof Substituting of $t=x \ln (n+\alpha)$ in (8), by calculation, we have

$$
\omega(n)=\int_{0}^{\infty} \frac{1}{(1+t)^{\lambda}} t^{\frac{\lambda}{2}-1} d t=B\left(\frac{\lambda}{2}, \frac{\lambda}{2}\right) .
$$

Since by the conditions and for fixed $x>0$

$$
h(x, y):=\frac{\ln ^{\frac{\lambda}{2}-1}(y+\alpha)}{(y+\alpha) \ln ^{\lambda} e(y+\alpha)^{x}}=\frac{\ln ^{\frac{\lambda}{2}-1}(y+\alpha)}{(y+\alpha)[1+x \ln (y+\alpha)]^{\lambda}}
$$

is strictly decreasing and strictly convex in $y \in\left(\frac{1}{2}, \infty\right)$, then by the Hermite-Hadamard inequality (cf. [20]), we find

$$
\begin{aligned}
& \varpi(x)<x^{\frac{\lambda}{2}} \int_{\frac{1}{2}}^{\infty} \frac{\ln ^{\frac{\lambda}{2}-1}(y+\alpha)}{(y+\alpha)[1+x \ln (y+\alpha)]^{\lambda}} d y \stackrel{t=x \ln (y+\alpha)}{=} \int_{x \ln \left(\frac{1}{2}+\alpha\right)}^{\infty} \frac{t^{\frac{\lambda}{2}-1}}{(1+t)^{\lambda}} d t \leq B\left(\frac{\lambda}{2}, \frac{\lambda}{2}\right), \\
& \varpi(x)>x^{\frac{\lambda}{2}} \int_{1}^{\infty} \frac{\ln ^{\frac{\lambda}{2}-1}(y+\alpha)}{(y+\alpha)[1+x \ln (y+\alpha)]^{\lambda}} d y \\
& \stackrel{t=x \ln (y+\alpha)}{=} \int_{x \ln (1+\alpha)}^{\infty} \frac{t^{\frac{\lambda}{2}-1} d t}{(1+t)^{\lambda}}=B\left(\frac{\lambda}{2}, \frac{\lambda}{2}\right)\left(1-\theta_{\lambda}(x)\right)>0, \\
& 0<\theta_{\lambda}(x):=\frac{1}{B\left(\frac{\lambda}{2}, \frac{\lambda}{2}\right)} \int_{0}^{x \ln (1+\alpha)} \frac{t^{\frac{\lambda}{2}-1}}{(1+t)^{\lambda}} d t<\frac{1}{B\left(\frac{\lambda}{2}, \frac{\lambda}{2}\right)} \int_{0}^{x \ln (1+\alpha)} t^{\frac{\lambda}{2}-1} d t=\frac{2[x \ln (1+\alpha)]^{\frac{\lambda}{2}}}{\lambda B\left(\frac{\lambda}{2}, \frac{\lambda}{2}\right)},
\end{aligned}
$$

that is, (10) is valid.

Lemma 2 Let the assumptions of Lemma 1 be fulfilled and, additionally, $0<p<1, \frac{1}{p}+\frac{1}{q}=$ $1, a_{n} \geq 0, n \in \mathbf{N}, f(x)$ is a non-negative measurable function in $(0, \infty)$. Then we have the following inequalities:

$$
\begin{aligned}
J & :=\left\{\sum_{n=1}^{\infty} \frac{\ln ^{\frac{p \lambda}{2}}-1}{n+\alpha}\left[\int_{0}^{\infty} \frac{f(x)}{\ln ^{\lambda} e(n+\alpha)^{x}} d x\right]^{p}\right\}^{\frac{1}{p}} \\
& \geq\left[B\left(\frac{\lambda}{2}, \frac{\lambda}{2}\right)\right]^{\frac{1}{q}}\left\{\int_{0}^{\infty} \varpi(x) x^{p\left(1-\frac{\lambda}{2}\right)-1} f^{p}(x) d x\right\}^{\frac{1}{p}},
\end{aligned}
$$




$$
\begin{aligned}
L_{1} & :=\left\{\int_{0}^{\infty} \frac{x^{\frac{q \lambda}{2}-1}}{[\varpi(x)]^{q-1}}\left[\sum_{n=1}^{\infty} \frac{a_{n}}{\ln ^{\lambda} e(n+\alpha)^{x}}\right]^{q} d x\right\}^{\frac{1}{q}} \\
& \geq\left\{B\left(\frac{\lambda}{2}, \frac{\lambda}{2}\right) \sum_{n=1}^{\infty}(n+\alpha)^{q-1} \ln ^{q\left(1-\frac{\lambda}{2}\right)-1}(n+\alpha) a_{n}^{q}\right\}^{\frac{1}{q}} .
\end{aligned}
$$

Proof By the reverse Hölder inequality (cf. [20]) and (10), it follows that

$$
\begin{aligned}
{\left[\int_{0}^{\infty} \frac{f(x) d x}{\ln ^{\lambda} e(n+\alpha)^{x}}\right]^{p} } & \left\{\int_{0}^{\infty} \frac{1}{\ln ^{\lambda} e(n+\alpha)^{x}}\left[\frac{x^{\left(1-\frac{\lambda}{2}\right) / q}}{\ln ^{\left(1-\frac{\lambda}{2}\right) / p}(n+\alpha)} \frac{f(x)}{(n+\alpha)^{\frac{1}{p}}}\right]\right. \\
& \left.\cdot\left[\frac{\ln ^{\left(1-\frac{\lambda}{2}\right) / p}(n+\alpha)}{x^{\left(1-\frac{\lambda}{2}\right) / q}}(n+\alpha)^{\frac{1}{p}}\right] d x\right\}^{p} \\
\geq & \int_{0}^{\infty} \frac{1}{\ln ^{\lambda} e(n+\alpha)^{x}} \frac{x^{\left(1-\frac{\lambda}{2}\right)(p-1)}}{(n+\alpha) \ln ^{1-\frac{\lambda}{2}}(n+\alpha)} f^{p}(x) d x \\
& \cdot\left\{\int_{0}^{\infty} \frac{(n+\alpha)^{q-1}}{\ln ^{\lambda} e(n+\alpha)^{x}} \frac{\ln ^{\left(1-\frac{\lambda}{2}\right)(q-1)}(n+\alpha)}{x^{1-\frac{\lambda}{2}}} d x\right\}^{p-1} \\
= & \left\{\omega(n)(n+\alpha)^{q-1} \ln ^{q\left(1-\frac{\lambda}{2}\right)-1}(n+\alpha)\right\}^{p-1} \\
& \cdot \int_{0}^{\infty} \frac{1}{\ln ^{\lambda} e(n+\alpha)^{x}} \frac{x^{\left(1-\frac{\lambda}{2}\right)(p-1)}}{(n+\alpha) \ln ^{1-\frac{\lambda}{2}}(n+\alpha)} f^{p}(x) d x \\
= & {\left[B\left(\frac{\lambda}{2}, \frac{\lambda}{2}\right)\right]^{p-1}(n+\alpha) \ln ^{1-\frac{p \lambda}{2}}(n+\alpha) } \\
& \cdot \int_{0}^{\infty} \frac{1}{\ln ^{\lambda} e(n+\alpha)^{x}} \frac{x^{\left(1-\frac{\lambda}{2}\right)(p-1)}}{(n+\alpha) \ln ^{1-\frac{\lambda}{2}}(n+\alpha)} f^{p}(x) d x .
\end{aligned}
$$

Then, by the Lebesgue term-by-term integration theorem ( $c f$. [21]), we have

$$
\begin{aligned}
J & \geq\left[B\left(\frac{\lambda}{2}, \frac{\lambda}{2}\right)\right]^{\frac{1}{q}}\left\{\sum_{n=1}^{\infty} \int_{0}^{\infty} \frac{1}{\ln ^{\lambda} e(n+\alpha)^{x}} \frac{x^{\left(1-\frac{\lambda}{2}\right)(p-1)} f^{p}(x) d x}{(n+\alpha) \ln ^{1-\frac{\lambda}{2}}(n+\alpha)}\right\}^{\frac{1}{p}} \\
& =\left[B\left(\frac{\lambda}{2}, \frac{\lambda}{2}\right)\right]^{\frac{1}{q}}\left\{\int_{0}^{\infty} \sum_{n=1}^{\infty} \frac{x^{\frac{\lambda}{2}}}{\ln ^{\lambda} e((n+\alpha))^{x}} \frac{x^{p\left(1-\frac{\lambda}{2}\right)-1} f^{p}(x) d x}{(n+\alpha) \ln ^{1-\frac{\lambda}{2}}(n+\alpha)}\right\}^{\frac{1}{p}} \\
& =\left[B\left(\frac{\lambda}{2}, \frac{\lambda}{2}\right)\right]^{\frac{1}{q}}\left\{\int_{0}^{\infty} \varpi(x) x^{p\left(1-\frac{\lambda}{2}\right)-1} f^{p}(x) d x\right\}^{\frac{1}{p}},
\end{aligned}
$$

and (11) follows. Still, by the reverse Hölder inequality, we have

$$
\begin{aligned}
& {\left[\sum_{n=1}^{\infty} \frac{a_{n}}{\ln ^{\lambda} e(n+\alpha)^{x}}\right]^{q}} \\
& \quad=\left\{\sum_{n=1}^{\infty} \frac{1}{\ln ^{\lambda} e(n+\alpha)^{x}}\left[\frac{x^{\left(1-\frac{\lambda}{2}\right) / q}}{\ln ^{\left(1-\frac{\lambda}{2}\right) / p}(n+\alpha)} \cdot \frac{1}{(n+\alpha)^{\frac{1}{p}}}\right]\left[\frac{\ln ^{\left(1-\frac{\lambda}{2}\right) / p}(n+\alpha)}{x^{\left(1-\frac{\lambda}{2}\right) / q}}(n+\alpha)^{\frac{1}{p}} a_{n}\right]\right\}^{q}
\end{aligned}
$$




$$
\begin{aligned}
\leq & \left\{\sum_{n=1}^{\infty} \frac{1}{\ln ^{\lambda} e(n+\alpha)^{x}} \frac{x^{\left(1-\frac{\lambda}{2}\right)(p-1)}}{(n+\alpha) \ln ^{1-\frac{\lambda}{2}}(n+\alpha)}\right\}^{q-1} \\
& \cdot \sum_{n=1}^{\infty} \frac{(n+\alpha)^{q-1}}{\ln ^{\lambda} e(n+\alpha)^{x}} \frac{\ln ^{\left(1-\frac{\lambda}{2}\right)(q-1)}(n+\alpha)}{x^{1-\frac{\lambda}{2}}} a_{n}^{q} \\
= & \frac{[\varpi(x)]^{q-1}}{x^{\frac{q \lambda}{2}}-1} \sum_{n=1}^{\infty} \frac{(n+\alpha)^{q-1}}{\ln ^{\lambda} e(n+\alpha)^{x}} x^{\frac{\lambda}{2}-1} \ln ^{\left(1-\frac{\lambda}{2}\right)(q-1)}(n+\alpha) a_{n}^{q} .
\end{aligned}
$$

Then, by the Lebesgue term-by-term integration theorem, we have

$$
\begin{aligned}
L_{1} & \geq\left\{\int_{0}^{\infty} \sum_{n=1}^{\infty} \frac{(n+\alpha)^{q-1}}{\ln ^{\lambda} e(n+\alpha)^{x}} x^{\frac{\lambda}{2}-1} \ln ^{\left(1-\frac{\lambda}{2}\right)(q-1)}(n+\alpha) a_{n}^{q} d x\right\}^{\frac{1}{q}} \\
& =\left\{\sum_{n=1}^{\infty}\left[\ln ^{\frac{\lambda}{2}}(n+\alpha) \int_{0}^{\infty} \frac{x^{\frac{\lambda}{2}-1} d x}{\ln ^{\lambda} e(n+\alpha)^{x}}\right](n+\alpha)^{q-1} \ln ^{q\left(1-\frac{\lambda}{2}\right)-1}(n+\alpha) a_{n}^{q}\right\}^{\frac{1}{q}} \\
& =\left\{\sum_{n=1}^{\infty} \omega(n)(n+\alpha)^{q-1} \ln ^{q\left(1-\frac{\lambda}{2}\right)-1}(n+\alpha) a_{n}^{q}\right\}^{\frac{1}{q}},
\end{aligned}
$$

and then in view of (10), inequality (12) follows.

\section{Main results}

In this paper, for $0<p<1(q<0)$, we still use the normal expressions $\|f\|_{p, \Phi}$ and $\|a\|_{q, \Psi}$. We also introduce two functions

$$
\begin{aligned}
& \Phi(x):=\left(1-\theta_{\lambda}(x)\right) x^{p\left(1-\frac{\lambda}{2}\right)-1} \quad(x>0) \quad \text { and } \\
& \Psi(n):=(n+\alpha)^{q-1} \ln ^{q\left(1-\frac{\lambda}{2}\right)-1}(n+\alpha) \quad(n \in \mathbf{N}),
\end{aligned}
$$

wherefrom $[\Phi(x)]^{1-q}=\left(1-\theta_{\lambda}(x)\right)^{1-q} x^{\frac{q \lambda}{2}-1}$ and $[\Psi(n)]^{1-p}=\frac{\ln \frac{p \lambda}{2}-1(n+\alpha)}{n+\alpha}$.

Theorem 1 If $0<\lambda \leq 2, \alpha \geq \frac{1}{2}, 0<p<1, \frac{1}{p}+\frac{1}{q}=1, f(x), a_{n} \geq 0,0<\|f\|_{p, \Phi}<\infty$ and $0<\|a\|_{q, \Psi}<\infty$, then we have the following equivalent inequalities:

$$
\begin{aligned}
I & :=\sum_{n=1}^{\infty} a_{n} \int_{0}^{\infty} \frac{f(x)}{\ln ^{\lambda} e(n+\alpha)^{x}} d x \\
& =\int_{0}^{\infty} f(x) \sum_{n=1}^{\infty} \frac{a_{n}}{\ln ^{\lambda} e(n+\alpha)^{x}} d x>B\left(\frac{\lambda}{2}, \frac{\lambda}{2}\right)\|f\|_{p, \Phi}\|a\|_{q, \Psi}, \\
J & =\left\{\sum_{n=1}^{\infty}[\Psi(n)]^{1-p}\left[\int_{0}^{\infty} \frac{f(x)}{\ln ^{\lambda} e(n+\alpha)^{x}} d x\right]^{p}\right\}^{\frac{1}{p}}>B\left(\frac{\lambda}{2}, \frac{\lambda}{2}\right)\|f\|_{p, \Phi}, \\
L & :=\left\{\int_{0}^{\infty}[\Phi(x)]^{1-q}\left[\sum_{n=1}^{\infty} \frac{a_{n}}{\ln ^{\lambda} e(n+\alpha)^{x}}\right]^{q} d x\right\}^{\frac{1}{q}}>B\left(\frac{\lambda}{2}, \frac{\lambda}{2}\right)\|a\|_{q, \Psi},
\end{aligned}
$$

where the constant $B\left(\frac{\lambda}{2}, \frac{\lambda}{2}\right)$ is best possible. 
Proof By the Lebesgue term-by-term integration theorem, there are two expressions for $I$ in (13). In view of (11), for $\varpi(x)>B\left(\frac{\lambda}{2}, \frac{\lambda}{2}\right)\left(1-\theta_{\lambda}(x)\right)$, we have (14). By the reverse Hölder inequality, we have

$$
I=\sum_{n=1}^{\infty}\left[\Psi^{\frac{-1}{q}}(n) \int_{0}^{\infty} \frac{1}{\ln ^{\lambda} e(n+\alpha)^{x}} f(x) d x\right]\left[\Psi^{\frac{1}{q}}(n) a_{n}\right] \geq J\|a\|_{q, \Psi} .
$$

Then by (14) we have (13). On the other hand, assuming that (13) is valid, setting

$$
a_{n}:=[\Psi(n)]^{1-p}\left[\int_{0}^{\infty} \frac{1}{\ln ^{\lambda} e(n+\alpha)^{x}} f(x) d x\right]^{p-1}, \quad n \in \mathbf{N},
$$

we obtain that $J^{p-1}=\|a\|_{q, \Psi}$. By (11), we find $J>0$. If $J=\infty$, then (14) is trivially valid; if $J<\infty$, then by (13) we have

$$
\begin{aligned}
& \|a\|_{q, \Psi}^{q}=J^{p}=I>B\left(\frac{\lambda}{2}, \frac{\lambda}{2}\right)\|f\|_{p, \Phi}\|a\|_{q, \Psi}, \quad \text { i.e., } \\
& \|a\|_{q, \Psi}^{q-1}=J>B\left(\frac{\lambda}{2}, \frac{\lambda}{2}\right)\|f\|_{p, \Phi},
\end{aligned}
$$

that is, (14) is equivalent to (13). In view of (12), for

$$
[\varpi(x)]^{1-q}>\left[B\left(\frac{\lambda}{2}, \frac{\lambda}{2}\right)\left(1-\theta_{\lambda}(x)\right)\right]^{1-q}
$$

we have (15). By the reverse Hölder inequality, we find

$$
I=\int_{0}^{\infty}\left[\Phi^{\frac{1}{p}}(x) f(x)\right]\left[\Phi^{\frac{-1}{p}}(x) \sum_{n=1}^{\infty} \frac{1}{\ln ^{\lambda} e(n+\alpha)^{x}} a_{n}\right] d x \geq\|f\|_{p, \Phi} L .
$$

Then by (15) we have (13). On the other hand, assuming that (13) is valid, setting

$$
f(x):=[\Phi(x)]^{1-q}\left[\sum_{n=1}^{\infty} \frac{1}{\ln ^{\lambda} e(n+\alpha)^{x}} a_{n}\right]^{q-1}, \quad x \in(0, \infty),
$$

we obtain that $L^{q-1}=\|f\|_{p, \Phi}$. By (12), we find $L>0$. If $L=\infty$, then (15) is trivially valid; if $L<\infty$, then by (13) we have

$$
\begin{aligned}
& \|f\|_{p, \Phi}^{p}=L^{q}=I>B\left(\frac{\lambda}{2}, \frac{\lambda}{2}\right)\|f\|_{p, \Phi}\|a\|_{q, \Psi}, \quad \text { i.e., } \\
& \|f\|_{p, \Phi}^{p-1}=L>B\left(\frac{\lambda}{2}, \frac{\lambda}{2}\right)\|a\|_{q, \Psi},
\end{aligned}
$$

that is, (15) is equivalent to (13). Hence, inequalities (13), (14) and (15) are equivalent.

$$
\text { For } 0<\varepsilon<\frac{p \lambda}{2} \text {, set } \widetilde{f}(x)=x^{\frac{\lambda}{2}+\frac{\varepsilon}{p}-1}, x \in(0,1) ; \widetilde{f}(x)=0, x \in[1, \infty) \text {, and }
$$

$$
\widetilde{a}_{n}=\frac{1}{n+\alpha} \ln ^{\frac{\lambda}{2}-\frac{\varepsilon}{q}-1}(n+\alpha), \quad n \in \mathbf{N} .
$$


If there exists a positive number $k\left(\geq B\left(\frac{\lambda}{2}, \frac{\lambda}{2}\right)\right)$ such that (13) is valid when replacing $B\left(\frac{\lambda}{2}, \frac{\lambda}{2}\right)$ with $k$, then, in particular, it follows that

$$
\begin{aligned}
\widetilde{I} & :=\sum_{n=1}^{\infty} \int_{0}^{\infty} \frac{1}{\ln ^{\lambda} e(n+\alpha)^{x}} \widetilde{a}_{n} \tilde{f}(x) d x>k\|\widetilde{f}\|_{p, \Phi}\|\widetilde{a}\|_{q, \Psi} \\
& =k\left\{\int_{0}^{1}\left(1-O\left(x^{\frac{\lambda}{2}}\right)\right) \frac{d x}{x^{-\varepsilon+1}}\right\}^{\frac{1}{p}}\left\{\frac{1}{(1+\alpha) \ln ^{\varepsilon+1}(1+\alpha)}+\sum_{n=2}^{\infty} \frac{1}{(n+\alpha) \ln ^{\varepsilon+1}(n+\alpha)}\right\}^{\frac{1}{q}} \\
& >k\left\{\frac{1}{\varepsilon}-O(1)\right\}^{\frac{1}{p}}\left\{\frac{1}{(1+\alpha) \ln ^{\varepsilon+1}(1+\alpha)}+\int_{1}^{\infty} \frac{d x}{(x+\alpha) \ln ^{\varepsilon+1}(x+\alpha)}\right\}^{\frac{1}{q}} \\
& =\frac{k}{\varepsilon}\{1-\varepsilon O(1)\}^{\frac{1}{p}}\left\{\frac{\varepsilon}{(1+\alpha) \ln ^{\varepsilon+1}(1+\alpha)}+\frac{1}{\ln ^{\varepsilon}(1+\alpha)}\right\}^{\frac{1}{q}} \\
\widetilde{I} & =\sum_{n=1}^{\infty} \frac{1}{n+\alpha} \ln ^{\frac{\lambda}{2}-\frac{\varepsilon}{q}-1}(n+\alpha) \int_{0}^{1} \frac{1}{\ln ^{\lambda} e(n+\alpha)^{x}} x^{\frac{\lambda}{2}+\frac{\varepsilon}{p}-1} d x \\
& t=x \ln (n+\alpha) \\
= & \sum_{n=1}^{\infty} \frac{1}{(n+\alpha) \ln ^{\varepsilon+1}(n+\alpha)} \int_{0}^{\ln (n+\alpha)} \frac{1}{(t+1)^{\lambda}} t^{\frac{\lambda}{2}+\frac{\varepsilon}{p}-1} d t \\
& \leq B\left(\frac{\lambda}{2}+\frac{\varepsilon}{p}, \frac{\lambda}{2}-\frac{\varepsilon}{p}\right)\left[\frac{1}{(1+\alpha) \ln ^{\varepsilon+1}(1+\alpha)}+\sum_{n=2}^{\infty} \frac{1}{(n+\alpha) \ln ^{\varepsilon+1}(n+\alpha)}\right] \\
& <B\left(\frac{\lambda}{2}+\frac{\varepsilon}{p}, \frac{\lambda}{2}-\frac{\varepsilon}{p}\right)\left[\frac{1}{(1+\alpha) \ln ^{\varepsilon+1}(1+\alpha)}+\int_{1}^{\infty} \frac{1}{(y+\alpha) \ln ^{\varepsilon+1}(y+\alpha)} d y\right] \\
& =\frac{1}{\varepsilon} B\left(\frac{\lambda}{2}+\frac{\varepsilon}{p}, \frac{\lambda}{2}-\frac{\varepsilon}{p}\right)\left[\frac{\varepsilon}{(1+\alpha) \ln ^{\varepsilon+1}(1+\alpha)}+\frac{1}{\ln ^{\varepsilon}(1+\alpha)}\right] .
\end{aligned}
$$

Hence by (18) and (19) it follows that

$$
\begin{gathered}
B\left(\frac{\lambda}{2}+\frac{\varepsilon}{p}, \frac{\lambda}{2}-\frac{\varepsilon}{p}\right)\left[\frac{\varepsilon}{(1+\alpha) \ln ^{\varepsilon+1}(1+\alpha)}+\frac{1}{\ln ^{\varepsilon}(1+\alpha)}\right] \\
>k\{1-\varepsilon O(1)\}^{\frac{1}{p}}\left\{\frac{\varepsilon}{(1+\alpha) \ln ^{\varepsilon+1}(1+\alpha)}+\frac{1}{\ln ^{\varepsilon}(1+\alpha)}\right\}^{\frac{1}{q}},
\end{gathered}
$$

and $B\left(\frac{\lambda}{2}, \frac{\lambda}{2}\right) \geq k\left(\varepsilon \rightarrow 0^{+}\right)$. Hence $k=B\left(\frac{\lambda}{2}, \frac{\lambda}{2}\right)$ is the best value of (13).

By the equivalence, the constant factor $B\left(\frac{\lambda}{2}, \frac{\lambda}{2}\right)$ in (14) and (15) is best possible. Otherwise we would reach a contradiction by (16) and (17) that the constant factor in (13) is not best possible.

Remark 1 (i) For $\lambda=1, \lambda_{1}=\lambda_{2}=\frac{1}{2}, \alpha=\frac{1}{2}$ in (13), (14) and (15), we have (7) and the following equivalent inequalities:

$$
\begin{aligned}
& \sum_{n=1}^{\infty} \frac{\ln ^{\frac{p}{2}-1}\left(n+\frac{1}{2}\right)}{n+\frac{1}{2}}\left[\int_{0}^{\infty} \frac{f(x)}{\ln e\left(n+\frac{1}{2}\right)^{x}} d x\right]^{p} \\
& >\pi^{p} \int_{0}^{\infty} \frac{1-\theta_{1}(x)}{x^{1-\frac{p}{2}}} f^{p}(x) d x,
\end{aligned}
$$


(ii) Setting $x=\frac{1}{\ln y}, g(y):=\frac{1}{y}(\ln y)^{\lambda-2} f\left(\frac{1}{\ln y}\right)$ and

$$
\phi(y):=\left(1-\theta_{\lambda}\left(\frac{1}{\ln y}\right)\right) y^{p-1}(\ln y)^{p\left(1-\frac{\lambda}{2}\right)-1} \quad(y \in(1, \infty))
$$

in (13), by simplifications, we find the following inequality with the homogeneous kernel:

$$
\begin{aligned}
& \sum_{n=1}^{\infty} a_{n} \int_{1}^{\infty} \frac{g(y)}{\ln ^{\lambda} y(n+\alpha)} d y \\
& \quad=\int_{1}^{\infty} g(y) \sum_{n=1}^{\infty} \frac{a_{n}}{\ln ^{\lambda} y(n+\alpha)} d x>B\left(\frac{\lambda}{2}, \frac{\lambda}{2}\right)\|g\|_{p, \phi}\|a\|_{q, \Psi} .
\end{aligned}
$$

It is evident that (22) is equivalent to (13), and then the constant factor $B\left(\frac{\lambda}{2}, \frac{\lambda}{2}\right)$ in (22) is still best possible. In the same way as in (14) and (15), we have the following inequalities equivalent to (13) with the best constant factor $B\left(\frac{\lambda}{2}, \frac{\lambda}{2}\right)$ :

$$
\begin{aligned}
& \left\{\sum_{n=1}^{\infty}[\Psi(n)]^{1-p}\left[\int_{1}^{\infty} \frac{g(y)}{\ln ^{\lambda} y(n+\alpha)} d y\right]^{p}\right\}^{\frac{1}{p}}>B\left(\frac{\lambda}{2}, \frac{\lambda}{2}\right)\|g\|_{p, \phi}, \\
& \left\{\int_{1}^{\infty}[\phi(y)]^{1-q}\left[\sum_{n=1}^{\infty} \frac{a_{n}}{\ln ^{\lambda} y(n+\alpha)}\right]^{q} d y\right\}^{\frac{1}{q}}>B\left(\frac{\lambda}{2}, \frac{\lambda}{2}\right)\|a\|_{q, \Psi} .
\end{aligned}
$$

\section{Competing interests}

The authors declare that they have no competing interests.

\section{Authors' contributions}

TL participated in the design of the study and performed the numerical analysis. BY carried out the mathematical studies, participated in the sequence alignment and drafted the manuscript. LH conceived of the study, and participated in its design and coordination. All authors read and approved the final manuscript.

\section{Author details}

${ }^{1}$ College of Mathematics and Statistics, Jishou University, Hunan, Jishou 416000, P.R. China. ${ }^{2}$ Department of Mathematics, Guangdong University of Education, Guangzhou, Guangdong 510303, P.R. China.

\section{Acknowledgements}

This work is supported by the National Natural Science Foundation of China (No. 61370186).

Received: 19 November 2013 Accepted: 6 February 2014 Published: 04 Mar 2014

\section{References}

1. Hardy, GH, Littlewood, JE, Pólya, G: Inequalities. Cambridge University Press, Cambridge (1934)

2. Mitrinović, DS, Pečarić, JE, Fink, AM: Inequalities Involving Functions and Their Integrals and Derivatives. Kluwer Academic, Boston (1991)

3. Yang, B: Hilbert-Type Integral Inequalities. Bentham Science Publishers, Sharjah (2009)

4. Yang, B: Discrete Hilbert-Type Inequalities. Bentham Science Publishers, Sharjah (2011)

5. Yang, B: An extension of Mulholland's inequality. Jordan J. Math. Stat. 3(3), 151-157 (2010)

6. Yang, B: On Hilbert's integral inequality. J. Math. Anal. Appl. 220, 778-785 (1998)

7. Yang, B: The Norm of Operator and Hilbert-Type Inequalities. Science Press, Beijing (2009) (in Chinese) 
8. Yang, B, Brnetić, I, Krnić, M, Pečarić, J: Generalization of Hilbert and Hardy-Hilbert integral inequalities. Math. Inequal Appl. 8(2), 259-272 (2005)

9. Krnić, M, Pečarić, J: Hilbert's inequalities and their reverses. Publ. Math. (Debr.) 67(3-4), 315-331 (2005)

10. Jin, J, Debnath, L: On a Hilbert-type linear series operator and its applications. J. Math. Anal. Appl. 371, 691-704 (2010)

11. Azar, L: On some extensions of Hardy-Hilbert's inequality and applications. J. Inequal. Appl. 2009, 546829 (2009)

12. Yang, B, Rassias, T: On the way of weight coefficient and research for Hilbert-type inequalities. Math. Inequal. Appl. 6(4), 625-658 (2003)

13. Arpad, B, Choonghong, O: Best constant for certain multilinear integral operator. J. Inequal. Appl. 2006, 28582 (2006)

14. Kuang, J, Debnath, L: On Hilbert's type inequalities on the weighted Orlicz spaces. Pac. J. Appl. Math. 1(1), 95-103 (2007)

15. Zhong, W: The Hilbert-type integral inequality with a homogeneous kernel of $\lambda$-degree. J. Inequal. Appl. 2008 $917392(2008)$

16. Li, Y, He, B: On inequalities of Hilbert's type. Bull. Aust. Math. Soc. 76(1), 1-13 (2007)

17. Yang, B: A mixed Hilbert-type inequality with a best constant factor. Int. J. Pure Appl. Math. 20(3), 319-328 (2005)

18. Yang, B: A half-discrete Hilbert's inequality. J. Guangdong Univ. Educ. 31(3), 1-7 (2011)

19. Yang, B: A half-discrete reverse Hilbert-type inequality with a homogeneous kernel of positive degree. J. Zhanjiang Norm. Coll. 32(3), 5-9 (2011)

20. Kuang, J: Applied Inequalities. Shangdong Science Technic Press, Jinan (2004) (in Chinese)

21. Kuang, J: Introduction to Real Analysis. Hunan Education Press, Chansha (1996) (in Chinese)

10.1186/1029-242X-2014-103

Cite this article as: Liu et al.: On a half-discrete reverse Mulholland-type inequality and an extension. Journal of Inequalities and Applications 2014, 2014:103

\section{Submit your manuscript to a SpringerOpen ${ }^{\circ}$ journal and benefit from:}

- Convenient online submission

- Rigorous peer review

- Immediate publication on acceptance

Open access: articles freely available online

- High visibility within the field

- Retaining the copyright to your article 